\title{
PRODUCCIÓN DE NÉCTAR EN MATORRALES DEL SUR DE ESPAÑA (ANDALUCÍA)
}

\author{
M.Isabel HIDALGO y Baltasar CABEZUDO
}

RESUMEN. Producción de néctar en matorrales del Sur de España (Andalucía). Con el fin de evaluar los recursos alimenticios de Apis mellifera, se ha realizado un estudio de la producciòn de néctar de las especies más representativas de un matorral desarrollado en la Sierra de Mijas (Málaga, S de España). La producción de azúcar por flor/especie se ha calculado en función del volumen de néctar y su concentración en azúcares. La producción de azúcar por $\mathrm{m}^{2} / \mathrm{especie} \mathrm{se} \mathrm{ha} \mathrm{calculado} \mathrm{en} \mathrm{función} \mathrm{de} \mathrm{la} \mathrm{producción} \mathrm{de} \mathrm{flores} \mathrm{por} \mathrm{m}^{2} /$ año. En 66 de las 115 especies estudiadas se ha detectado la presencia de néctar, cuantificándose la cantidad de azúcar en 49 de ellas.

Palabras clave. Melitopalinología, néctar floral, Andalucía, España

ABSTRACT. Nectar production in scrubland of southern Spain (Andalusia). The aim of this work is to evaluate the alimentary resources for the Apis mellifera. A study has been carried out about the nectar production of the most representative species of a scrubland in Sierra de Mijas (Málaga, Spain). The sugar production per flower species has been calculated based on the nectar volume and its sugar concentration and the sugar production per $\mathrm{m}^{2} / \mathrm{species}$ according to the flower production per $\mathrm{m}^{2} /$ year. The nectar presence has been detected in 66 out of the 115 studied species and the sugar amount has been quantified in 49 of them.

Key words: Melissopalynogy, floral nectar, Andalusia, Spain.

\section{INTRODUCCIÓN}

El néctar producido por las flores constituye uno de los principales recursos alimenticios de la abeja (Apis mellifera), ya que es utilizado por estos insectos como materia prima para la producción de miel. En este sentido, el comportamiento de la abeja va a estar muy relacionado con la cantidad y concentración de azúcares presentes en el néctar de cada tipo de flor (Percival, 1961; Corbet, 1978; Corbet et al.,1979a,b,1984). La cuantificación y cualificación del néctar en una determinada especie vegetal resulta de gran interés en los estudios de biología floral, y más concretamente en el campo de los estudios melitopalinológicos, donde los objetivos principales estarían encaminados a determinar el interés, como materia prima de miel, de dichas especies en un territorio determinado.

Los trabajos sobre productividad floral se han realizado básicamente en las dos últimas décadas, siendo más frecuentes los relacionados 
con la producción de néctar (Nuñez,1977; Bond \& Brown,1979; Corbet et al.,1979 a, b; Willmer,1980; Pflumm, 1985, etc.). En la zona mediterránea en general y en Andalucía en particular, son muy recientes los trabajos desarrollados en este sentido, destacando entre otros los de Weber El-Ghobary (1984), Fernández et al.(1989), Herrera (1985a,b), Devesa et al. (1985), Muñoz y Devesa (1987), Talavera et al. (1988) y Ortiz (1994). Otros trabajos, más completos desde el punto de vista de aprovechamiento apícola, incluyen los parámetros de producción de flores por $\mathrm{m}^{2}$ de cada especie y cobertura vegetal, lo que permite una mayor aproximación a la valoración de la potencialidad melífera de una zona (Ortiz, 1991).

El objetivo de este trabajo se centra en la determinación del volumen y concentración de azúcares del néctar de las especies más características del matorral desarrollado en la Sierra de Mijas (Málaga). Estos datos, junto a los referidos a fenología de la floración, cobertura vegetal y producción de flores por metro cuadrado de las mismas especies (Hidalgo y Cabezudo, 1994 y 1995), nos aportan nuevos valores en cuanto a productividad melífera del matorral en el área de estudio y su uso como recurso alimenticio para Apis mellifera.

\section{MATERIAL Y MÉTODO}

La Sierra de Mijas se encuentra situada en la provincia de Málaga (España), climatológicamente se encuadra en la región Mediterránea con épocas de lluvia en otoñoinvierno y máxima insolación en verano. El período de mayor aridez se sitúa desde finales de Mayo hasta finales de Septiembre. La temperatura media anual es de $17,5^{\circ} \mathrm{C}$ con medias máximas de $25,6^{\circ} \mathrm{C}$ en Agosto y medias mínimas de $11,1^{\circ} \mathrm{C}$ en Enero; la precipitación media anual es de $610 \mathrm{~mm}$.
Desde un punto de vista biogeográfico la zona estudiada pertenece a la Región Mediterránea, y en función de sus peculiaridades florísticas y fitosociológicas (vegetación), se incluye, junto con territorios próximos, en el Subsector Mijense del Sector Rondeño. La vegetación está representada por un coscojar-encinar perteneciente a la asociación Rhamno velutini-Quercetum cocciferae, formada por bosquetes de fanerófitos y nanofanerófitos que colonizan suelos sobre mármoles dolomíticos. El jaralaulagar, predominante en la Sierra de Mijas, pertenece a la asociación Cisto clussii-ulicetum baetici.

Se ha cuantificado la cantidad de néctar y concentración en azúcar en 115 especies leñosas y herbáceas consideradas de interés melífero en la zona. Dicha selección se ha realizado en base a las observaciones de campo previas y a los datos que figuran en la bibliografía referentes a géneros o especies presentes en la zona.

Siguiendo la metodología empleada por Herrera (1985a,b), se han cortado ramas floríferas de varios individuos de una misma especie durante el periodo en el que presentaron máxima floración. Las ramas se han colocado en bolsas de plástico transparentes durante 24 horas, en condiciones de luz natural y temperatura ambiente. Posteriormente las flores se han observado bajo una lupa binocular para detectar la presencia de néctar. En aquellas especies en las que se detectó, éste fue extraído mediante una micropipeta capilar de $5 \mu$ l. De esta manera, el volumen de néctar se ha calculado midiendo con una regla la longitud de la columna de néctar resultante y relacionándolo con el volumen equivalente en $\mu l$ (Cruden y Hermann,1983).

Debido a la gran fragilidad y corta duración de las flores de Cistus spp., éstas se han embolsado directamente en el campo con una malla de nailon (Herrera 1987a; Ortiz, 1991). Según varios autores (Brandt \& 
Gottsberger, 1988; Bosh, 1992), las flores de estas especies se abren por la mañana y pierden los pétalos a primeras horas de la tarde. Por ello se han embolsado por la tarde varios botones florales, realizándose la medición de néctar al día siguiente entre las 9 y 10 de la mañana sobre las flores ya abiertas.

En las flores de algunas especies, la extracción de néctar no ha sido posible debido al tamaño de la flor y a su localización (p.e. Calendula arvensis). En otros casos, se ha detectado la presencia de néctar mediante las observaciones de campo o bien con lupa binocular, pero no se ha podido cuantificar por ser éste muy escaso (p.e. Diplotaxis virgata).

La cuantificación del volumen de néctár se ha llevado a cabo en varias flores de las ramas procedentes de distintos individuos. Por lo general, en cada extracción de néctar se ha medido seguidamente la concentración de sólidos disueltos, siendo los azúcares (sacarosa, glucosa y fructosa) los que representan la mayor parte de éstos (Percival,1961). La concentración de azúcares se ha medido mediante dos refractómetros (0-32\% y 28-62\%) marca Shibuja de alto contraste y de una precisión del $0,2 \%$. La lectura directa del refractómetro da la concentración en ${ }^{0}$ Brix, unidad de medida estándar empleada en la industria azucarera, y que es equivalente a $g$ de soluto por $100 \mathrm{~g}$ de disolución (Bolten et al. 1979). En el empleo de este método hay que tener en cuenta que se produce una sobrestimación de los valores de azúcar en un $8-11 \%$, ya que los aminoácidos contribuyen significativamente al índice de refracción (Inouye et al. 1980).

La cantidad de azúcar por flor se ha calculado a partir de los datos de concentración y volumen del néctar, según la fórmula propuesta por Cruden \& Hermann (1983): $\mathbf{P}=$ $\mathbf{V} \times \mathbf{C} \times \mathbf{Y}$, donde $\mathbf{P}=$ cantidad de azúcar (en peso), $. \mathbf{V}=$ volumen de néctar, $\mathbf{C}=$ concentración del néctar en "Brix. y $\mathbf{Y}=0,0046$ x $\mathbf{C}+0,9946$.

Este método de cuantificación del volumen y concentración de néctar es el habitualmente utilizado por diversos autores en trabajos similares (Herrera, 1985a,b; Devesa et al.,1985; Talavera et al.,1988; Ortiz,1994), lo que permite una comparación posterior de resultados.

La particularidad de usar ramas floríferas embolsadas se debe a que las mediciones efectuadas directamente en el campo pueden resultar con un alto porcentaje de concentración de azúcares (más del 60\%) y poco volumen de néctar, lo que dificulta enormemente la toma de datos y su observación. Este hecho ha sido puesto de manifiesto por Herrera (1985b), quien además comprobó que el producto de $\mathbf{C ~ x ~ V ~}$ (producción de azúcar) de varias especies de matorral, cortadas y embolsadas 24 horas, comparado con las mismas especies en el campo (protegidas 24 horas de los insectos), no resultó significativamente diferente, si bien el porcentaje de azúcar en el néctar de las flores cortadas es menor (no superior al 25\%), lo que se compensa con un mayor volumen que en las flores del campo. Este último hecho puede estar en relación con la humedad que proporciona la bolsa (Corbet et al.,1979b).

Finalmente, la producción de néctar por $\mathrm{m}^{2} /$ año de cada especie se ha estimado teniendo en cuenta el número de flores que produce cada especie por unidad de superficie $(\mathrm{m} 2)$ durante su periodo fenológico (Hidalgo y Cabezudo , 1995).

\section{RESULTADOS Y DISCUSIÓN}

En la tabla 1 se presentan los datos correspondientes a la cuantificación del néctar de las especies estudiadas y su reconversión en producción de azúcar. De un total de 115 especies, 49 resultaron carentes de secreción nectarífera. Estas especies suelen corresponder a familias como Cistaceae, Geraniaceae, Oleaceae y Ranunculaceae.En ninguna de las especies de Cistaceae se ha detectado la 


\begin{tabular}{|c|c|c|c|c|c|}
\hline $\begin{array}{l}\text { FAMILIAS } \\
\text { Especies } \\
\end{array}$ & V néctar & $\begin{array}{c}\mathrm{C} \\
\left({ }^{\circ} \text { Brik) }\right. \\
\end{array}$ & $\begin{array}{l}\text { P azúcar/flor } \\
(\mu \mathrm{g} / \text { flor/día })\end{array}$ & $\begin{array}{l}\text { P azúcar } / \mathbf{m}^{2} \\
\left(\mathbf{m g} / \mathbf{m}^{2} / \mathbf{a n ̃ o}\right)\end{array}$ & $\mathbf{n}$ \\
\hline \multicolumn{6}{|l|}{ AMARYLLIDACEAE } \\
\hline Leucojum autumnale & - & & & & 15 \\
\hline *Scilla autumnalis & - & & & & 12 \\
\hline \multicolumn{6}{|l|}{ ANACARDIACEAE } \\
\hline *Pistacia lentiscus (f) & - & & & & 13 \\
\hline *Pistacia terebinthus (f) & - & & & & 15 \\
\hline \multicolumn{6}{|l|}{ APIACEAE } \\
\hline Ferula communis & $3,31 \pm 0,68$ & $7,6 \pm 1,34$ & 259 & 46514 & 30 \\
\hline Thapsia villosa & $0,56 \pm 0,10$ & $11 \pm 0,54$ & 64 & 869 & 15 \\
\hline \multicolumn{6}{|l|}{ APOCYNACEAE } \\
\hline *Vinca difformis & $3,48 \pm 0,91$ & & 771 & & 25 \\
\hline \multicolumn{6}{|l|}{ ARECACEAE } \\
\hline Chamaerops humilis (m) & - & & & & 15 \\
\hline \multicolumn{6}{|l|}{ ASTERACEAE } \\
\hline Calendula arvensis & + & & & & 10 \\
\hline Carlina corymbosa & + & & & & 12 \\
\hline Carthamus arborescens & $2,06 \pm 0,42$ & $14 \pm 1,8$ & 305 & 956 & 15 \\
\hline${ }^{*}$ Centaurea sempervirens & $1,12 \pm 0,41$ & $18,2 \pm 2,6$ & 220 & & 25 \\
\hline Dittrichia viscosa & $0,22 \pm 0,12$ & $44 \pm 1,29$ & 116 & 8904 & 15 \\
\hline Galactites tomentosa & + & & & & 15 \\
\hline Helichrisum stoechas &. & & & & 12 \\
\hline Klasea flavescens & + & & & & 12 \\
\hline Ptilostemon hispanicus & $3,57 \pm 0,67$ & $27,12 \pm 3$ & 1084 & 30812 & 25 \\
\hline Scolymus hispanicus & + & & & & 16 \\
\hline *Silybum marianum & $0,67 \pm 0,1$ & $21,3 \pm 1,21$ & 156 & & 25 \\
\hline \multicolumn{6}{|l|}{ BORRAGINACEAE } \\
\hline Anchusa azurea & $3,25 \pm 0,41$ & $19 \pm 1,29$ & 668 & 4369 & 25 \\
\hline Borrago officinalis & $3,65 \pm 0,91$ & $15 \pm 1,09$ & 582 & 2646 & 25 \\
\hline Cynoglossum cheirifolium & $7,13 \pm 1,21$ & & 1570 & 7969 & 17 \\
\hline Cynoglossum creticum & $4,24 \pm 0,69$ & $15,2 \pm 1,78$ & 686 & 3018 & 25 \\
\hline
\end{tabular}

Tabla 1. Volumen de néctar por flor $(\mathrm{V})$, concentración $(\mathrm{C})$ y producción de azúcar $(\mathrm{P})$ por flor y por $\mathrm{m}^{2}$. En Asteraceae y Lavandula stoechas, los datos de producción de azúcar se refieren a la inflorescencia. Presencia de néctar sin cuantificar $(+)$; sin néctar $(-)$; producción de azúcar por flor $(*) ; \mathrm{n}^{\circ}$ de muestra estudiada $(\mathrm{n})$. Nectar volume per flower $(V)$, concentration $(C)$ and sugar produduction $(P)$ per flower and $\mathrm{m}^{2}$. In Asteraceae and Lavandula stoechas the production data are referred to the inflorescence. Nectar presence without quantification (+); without nectar (-); sugar production per flower $(*)$; sample studied number $(n)$. 


\begin{tabular}{|c|c|c|c|c|c|}
\hline $\begin{array}{l}\text { FAMILIAS } \\
\text { Especies } \\
\end{array}$ & V néctar & $\begin{array}{c}\text { C } \\
\left({ }^{\circ} \text { Brik }\right) \\
\end{array}$ & $\begin{array}{l}\text { P azúcar/flor } \\
(\mu \mathrm{g} / \text { flor/día })\end{array}$ & $\begin{array}{l}\text { P azúcar/m² } \\
\left(\mathbf{m g} / \mathbf{m}^{2} / \mathbf{a n ̃}\right)\end{array}$ & $\mathbf{n}$ \\
\hline \multicolumn{6}{|l|}{ BORRAGINACEAE } \\
\hline Echium albicans & $2,75 \pm 0,60$ & & 403 & 5899 & 25 \\
\hline *Echium creticum & $2,35 \pm 0,33$ & $12,77 \pm 1,7$ & 316 & & 20 \\
\hline Echium plantagineum & $2,92 \pm 0,67$ & $11,6 \pm 1,61$ & 355 & 9328 & 24 \\
\hline \multicolumn{6}{|l|}{ BRASSICACEAE } \\
\hline Alyssum serpyllifolium & - & & & & 13 \\
\hline *Biscutella auriculata & - & & & & 12 \\
\hline *Diplotaxis erucoides & + & & & & 15 \\
\hline Diplotaxis virgata & + & & & & 12 \\
\hline Eruca sativa & + & & & & 12 \\
\hline *Erysimum rondae & + & & & & 12 \\
\hline *Lobularia maritima & + & & & & 13 \\
\hline *Mathiola tristis & - & & & & 12 \\
\hline \multicolumn{6}{|l|}{ CAESALPINIACEAE } \\
\hline Ceratonia siliqua (f) & - & & & & 10 \\
\hline \multicolumn{6}{|l|}{ CAMPANULACEAE } \\
\hline *Campanula erimus & + & & & & 14 \\
\hline \multicolumn{6}{|l|}{ CAPRIFOLIACEAE } \\
\hline Lonicera implexa & $4,08 \pm 0,75$ & $18,4 \pm 2,1$ & 810 & 707 & 25 \\
\hline \multicolumn{6}{|l|}{ CARYOPHYLACEAE } \\
\hline *Arenaria modesta & - & & & & 10 \\
\hline Silene colorata & $11,5 \pm 5,3$ & $30,5 \pm 4,6$ & 3974 & 6780 & 5 \\
\hline \multicolumn{6}{|l|}{ CISTACEAE } \\
\hline Cistus albidus & - & & & & 11 \\
\hline Cistus clussii & - & & & & 10 \\
\hline Cistus crispus & - & & & & 10 \\
\hline Cistus monspeliensis & - & & & & 11 \\
\hline Cistus salvifolius & - & & & & 11 \\
\hline *Fumana ericoides & - & & & & 10 \\
\hline *Fumana laevipes & - & & & & 12 \\
\hline Fumana thymifolia & - & & & & 6 \\
\hline Halimium atriplicifolium & - & & & & 11 \\
\hline Halimium halimifolium & - & & & & 10 \\
\hline Helianthemum cinereum & - & & & & 6 \\
\hline Helianthemum croceum & - & & & & 5 \\
\hline
\end{tabular}

Tabla 1. (continuación). 


\begin{tabular}{|c|c|c|c|c|c|}
\hline $\begin{array}{l}\text { FAMILIAS } \\
\text { Especies } \\
\end{array}$ & V néctar & $\begin{array}{c}\mathrm{C} \\
\left({ }^{\circ} \text { Brik }\right) \\
\end{array}$ & $\begin{array}{l}\text { P azúcar/flor } \\
(\mu \mathrm{g} / \text { flor/día })\end{array}$ & $\begin{array}{l}\text { P azúcar/m² } \\
\left(\mathrm{mg} / \mathrm{m}^{2} / \mathbf{a n ̃ o}\right)\end{array}$ & $\mathbf{n}$ \\
\hline CISTACEAE & & & & & \\
\hline Helianthemum syriacum & - & & & & 7 \\
\hline *Helianthemum marifolium & - & & & & 6 \\
\hline *Helianthemum origanifolium & - & & & & 7 \\
\hline *Helianthemum sallicifolium & - & & & & 7 \\
\hline *Tuberaria guttata & - & & & & 8 \\
\hline CONVOLVULACEAE & & & & & \\
\hline *Convolvulus althaeoides & $1,9 \pm 0,42$ & $22 \pm 1,81$ & 458 & & 15 \\
\hline Convolvulus lanuginosus & + & & & & 12 \\
\hline CRASSULACEAE & & & & & \\
\hline *Sedum sediforme & $2,4 \pm 1,57$ & $30,4 \pm 2,2$ & 828 & & 18 \\
\hline DIPSACACEAE & & & & & \\
\hline$*$ Cephalaria leucanta & $0,84 \pm 0,36$ & $31 \pm 2,36$ & 296 & & 30 \\
\hline *Scabiosa atropurpurea & $3,37 \pm 0,73$ & $26 \pm 1,8$ & 976 & & 30 \\
\hline EUPHORBIACEAE & & & & & \\
\hline *Mercurialis tomentosa & + & & & & 10 \\
\hline FABACEAE & & & & & \\
\hline Anthyllis cytisoides & $1,17 \pm 0,25$ & $15,1 \pm 0,83$ & 188 & 6037 & 30 \\
\hline Calicotome villosa & + & & & & 10 \\
\hline Coronilla juncea & - & & & & 6 \\
\hline *Lupinus angustifolius & - & & & & 7 \\
\hline Ononis natrix & - & & & & 7 \\
\hline *Ononis viscosa & - & & & & 7 \\
\hline Psoralea bituminosa & $1,19 \pm 0,56$ & $21,3 \pm 1,5$ & 277 & 343 & 25 \\
\hline Ulex baeticus & - & & & & 10 \\
\hline FAGACEAE & & & & & \\
\hline Quercus coccifera $(\mathrm{m}, \mathrm{f})$ & - & & & & 6 \\
\hline Quercus rotundifolia $(\mathrm{m}, \mathrm{f})$ & - & & & & 6 \\
\hline GENTIANACEAE & & & & & \\
\hline *Centaurium erythraea & - & & & & 10 \\
\hline GERIANIACEAE & & & & & \\
\hline *Erodium aethiopicum & - & & & & 10 \\
\hline *Erodium primulaceum & - & & & & 10 \\
\hline
\end{tabular}

Tabla 1. (continuación). 


\begin{tabular}{|c|c|c|c|c|c|}
\hline $\begin{array}{l}\text { FAMILIAS } \\
\text { Especies }\end{array}$ & V néctar & $\begin{array}{c}\mathbf{C} \\
\left({ }^{\circ} \text { Brik }\right)\end{array}$ & $\begin{array}{l}\text { P azúcar/flor } \\
(\mu \mathrm{g} / \text { flor/día })\end{array}$ & $\begin{array}{l}\text { P azúcar/m² } \\
\left(\mathbf{m g} / \mathbf{m}^{2} / \mathbf{a n ̃ o}\right)\end{array}$ & $\mathbf{n}$ \\
\hline \multicolumn{6}{|l|}{ LAMIACEAE } \\
\hline Ballota hirsuta & $1,48 \pm 0,48$ & $25 \pm 1,7$ & 410 & 665 & 25 \\
\hline${ }^{*}$ Calamintha sylvatica & $1,53 \pm 0,31$ & $43 \pm 2,1$ & 784 & & 25 \\
\hline Lavandula stoechas & $0,61 \pm 0,1$ & $14,8 \pm 0,78$ & 96 & 150561 & 15 \\
\hline *Nepeta tuberosa & $3,08 \pm 0,22$ & $23,4 \pm 1,33$ & 794 & & 20 \\
\hline Phlomis lychnitis & $2,8 \pm 0,17$ & $36,8 \pm 3,38$ & 1199 & 685 & 25 \\
\hline Phlomis purpurea & $0,56 \pm 0,12$ & $36,5 \pm 6,8$ & 238 & 198 & 20 \\
\hline Rosmarinus officinalis & $14,6 \pm 3,71$ & & 2856 & 89970 & 50 \\
\hline Salvia verbenaca & $2,35 \pm 0,53$ & $16 \pm 3,33$ & 402 & 3769 & 25 \\
\hline Teucrium fruticans & $2,35 \pm 0,48$ & $18,6 \pm 0,74$ & 472 & 892 & 25 \\
\hline Teucrium lusitanicum & $0,1 \pm 0,09$ & $24 \pm 2,3$ & 26 & 1893 & 25 \\
\hline *Teucrium pseudochamaepitys & $1,28 \pm 0,31$ & $20,8 \pm 0,89$ & 290 & & 30 \\
\hline Thymbra capitata & $0,28 \pm 0,12$ & $23 \pm 1$ & 71 & 2321 & 25 \\
\hline Thymus mastichina & $0,56 \pm 0,21$ & $17 \pm 1,6$ & 102 & 3667 & 25 \\
\hline \multicolumn{6}{|l|}{ LILIACEAE } \\
\hline *Allium roseum & $3,3 \pm 0,60$ & $10,6 \pm 0,74$ & 365 & & 15 \\
\hline Asphodelus albus & $4,75 \pm 0,25$ & $11,7 \pm 3,93$ & 583 & 1125 & 15 \\
\hline *Asphodelus ramosus & $9 \pm 1,2$ & $12,8 \pm 1,45$ & 1214 & & 20 \\
\hline *Urginea maritima & - & & & & 10 \\
\hline \multicolumn{6}{|l|}{ LINACEAE } \\
\hline Linum suffruticosum & - & & & & 10 \\
\hline \multicolumn{6}{|l|}{ MALVACEAE } \\
\hline *Lavatera cretica & $2,62 \pm 0,61$ & $13 \pm 2,1$ & 359 & & 15 \\
\hline Malva hispanica & $3,6 \pm 0,71$ & $11 \pm 1,06$ & 414 & 184 & 20 \\
\hline \multicolumn{6}{|l|}{ MYRTACEAE } \\
\hline Myrtus communis & - & & & & 10 \\
\hline \multicolumn{6}{|l|}{ OLEACEAE } \\
\hline Olea europaea & - & & & & 10 \\
\hline Olea europaea var. sylvestris & - & & & & 10 \\
\hline \multicolumn{6}{|l|}{ ORCHIDACEAE } \\
\hline *Serapias parviflora & $1,4 \pm 0,72$ & $11,3 \pm 2,16$ & 166 & & 20 \\
\hline \multicolumn{6}{|l|}{ OROBANCHACEAE } \\
\hline *Orobanche ramosa & $2,8 \pm 1,14$ & $15,6 \pm 2,19$ & 466 & & 15 \\
\hline
\end{tabular}

Tabla 1. (continuación). 


\begin{tabular}{|c|c|c|c|c|c|}
\hline $\begin{array}{l}\text { FAMILIAS } \\
\text { Especies } \\
\end{array}$ & V néctar & $\begin{array}{c}\mathrm{C} \\
\left({ }^{\circ} \mathrm{Brik}\right) \\
\end{array}$ & $\begin{array}{l}\text { P azúcar/flor } \\
(\mu g / \text { flor/día) }\end{array}$ & $\begin{array}{l}\text { P azúcar/m² } \\
\left(\mathrm{mg} / \mathrm{m}^{2} / \mathbf{a n ̃ o}\right)\end{array}$ & $\mathbf{n}$ \\
\hline \multicolumn{6}{|l|}{ OXALICACEAE } \\
\hline Oxalis pes-caprae & $3 \pm 0,45$ & $6,4 \pm 0,8$ & 197 & 1080 & 25 \\
\hline \multicolumn{6}{|l|}{ PLUMBAGINACEAE } \\
\hline$*$ Armeria alliacea & $0,84 \pm 0,26$ & $30,3 \pm 1,89$ & 289 & & 25 \\
\hline \multicolumn{6}{|l|}{ PRIMULACEAE } \\
\hline${ }^{*}$ Coris monspeliensis & $2,07 \pm 0,25$ & $15,4 \pm 0,54$ & 340 & & 25 \\
\hline \multicolumn{6}{|l|}{ RANUNCULACEAE } \\
\hline Anemone palmata & - & & & & 11 \\
\hline Clematis flammula & - & & & & 10 \\
\hline *Delphinium pentagynum & - & & & & 8 \\
\hline *Ranunculus gramineus & - & & & & 10 \\
\hline \multicolumn{6}{|l|}{ RHAMNACEAE } \\
\hline Rhamnus velutinus (f) & + & & & & 10 \\
\hline \multicolumn{6}{|l|}{ ROSACEAE } \\
\hline Prunus dulcis & $17,8 \pm 5,84$ & & 20435 & 7187 & 25 \\
\hline Prunus persica & $2,4 \pm 0,77$ & $6,87 \pm 1,22$ & 169 & 417 & 25 \\
\hline *Rosa canina & - & & & & 8 \\
\hline Rubus ulmifolius & - & & & & 8 \\
\hline \multicolumn{6}{|l|}{ SMILACEAE } \\
\hline Smilax aspera (f) & $0,58 \pm 0,06$ & $42 \pm 1,5$ & 289 & 621 & 15 \\
\hline \multicolumn{6}{|l|}{ THYMELEACEAE } \\
\hline Thymelaea hirsuta & + & & & & 10 \\
\hline \multicolumn{6}{|l|}{ VALERIANACEAE } \\
\hline Fedia cornucopiae & + & & & & 10 \\
\hline
\end{tabular}

Tabla 1. (continuación).

presencia de néctar, factor que sí ha sido cuantificado por Ortiz (1994) en especies del género Cistus . Probablemente la mayor sequía de nuestra zona, en comparación con la zona de estudio de dicho autor, ha limitado la secreción de néctar en estas especies. Otra posible explicación, según han observado algunos autores en Cistus ladanifer (Talavera et. al, 1993), la podríamos encontrar en que la secreción fuera escasa al comienzo de la antesis, esto es, a primera hora de la mañana, periodo durante el cual se efectuaron las mediciones.

El volumen de néctar por flor ha oscilado entre $0,1 \mu \mathrm{l} /$ flor/día (Teucrium lusitanicum) y 17,8 $\mu \mathrm{l} /$ flor/día (Prunus dulcis). Otras especies con valores máximos de néctar han sido Rosmarinus officinalis $(14,6)$, Silene colorata $(11,5)$ y Asphodelus ramosus $(9,4)$. La 


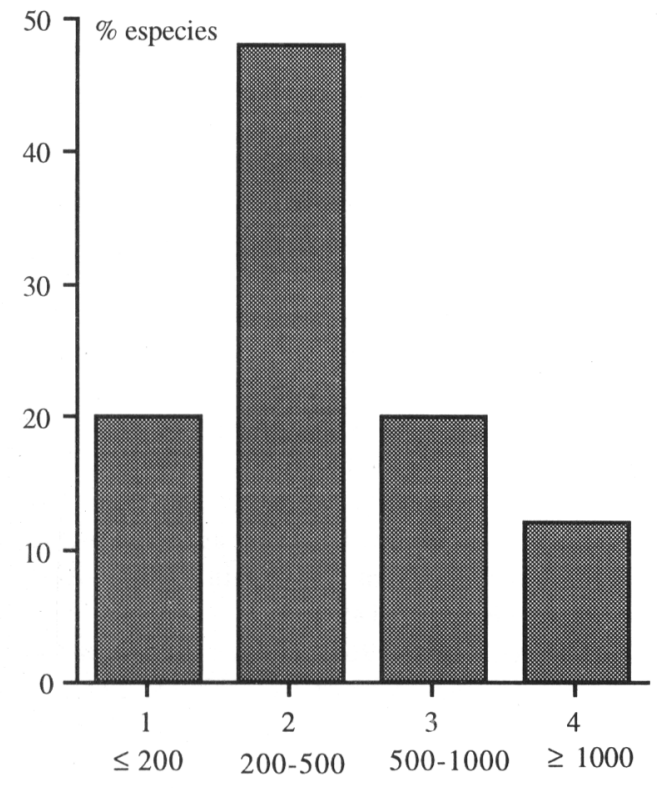

Producción de azúcar / flor ( $\mu$ g/flor/día)

Figura 1. Clases de frecuencia de las especies estudiadas en función de la producción de azúcar por flor y día (mg/flor/día). Frequences classes of the studied species according to the sugar production per flower and day (mg/flower/day).

concentración del néctar ha variado desde 6,4 ${ }^{0} \mathrm{Brik}$ (Oxalis pres-caprae) hasta $44{ }^{0} \mathrm{Brik}$ (Dittrichia viscosa). Hemos aplicado, para ver la correlación entre volumen y concentración, el test de rango de Spearman. Dicho test ha resultado significativo y negativo para un $\alpha=$ 0,05, lo cual indica que a mayor volumen de néctar la concentración es menor, relación inversa puesta de manifiesto por Corbet et al. (1979 a,b).

La producción de azúcar/flor varía desde $26 \mu \mathrm{g} / \mathrm{flor} / \mathrm{día}$ (Teucrium lusitanicum) hasta $2.035 \mu \mathrm{g} /$ flor/día (Prunus dulcis). Estos resultados de producción de azúcar/flor son aproximados a los obtenidos por otros autores en zonas de Andalucía Occidental (Herrera, 1985; Talavera et al., 1988; Ortiz, 1991). Las diferencias que puedan existir podrían deberse a los diferentes factores internos y externos que influyen en la secreción nectarífera (ver Shuel, 1975).

En la figura 1 se representa cuatro clases de frecuencia en donde se agrupan las especies cuyo néctar ha podido ser cuantificado y las cuales se han clasificado según su producción de azúcar por flor. La clase más frecuente, con un $48 \%$ de las especies, comprende especies que producen entre $200-500 \mu \mathrm{g}$. A la clase con una producción mayor de $1000 \mu \mathrm{g} /$ flor pertenecen sólo unas pocas especies, tales como Rosmarinus officinalis, Prunus dulcis, Cynnoglossum cheirifolium etc.

La producción de azúcar en $\mathrm{mg} / \mathrm{m}^{2} / \mathrm{año}$ (tab. 1) presenta valores máximos en las especies Lavandula stoechas (150.561), Rosmarinus officinalis (89.970), Prunus dulcis (7.187) y Ferula communis (46.514).

En 17 especies de las estudiadas se ha detectado la presencia de néctar (+), pero este no ha podido ser cuantificado mediante la metodología aplicada. Estas especies pertenecen generalmente a familias como Asteraceae y Brassicaceae.

\section{BIBLIOGRAFIA}

BOLTEN, A.B., P. FEINSINGER, H.G. HERBERT y I. BAKER -1979- On the calculation of sugar concentration in flower nectar. Oecologia, 41: 301-304.

BOND, H.W. y W.L. BROWN -1979- The explotation of floral nectar in Eucalyptus incrassata by honeyeaters and honeybees. Oecologia, 44: 105-111.

BOSCH, J. -1992- Floral biology and pollinators of three co-occurring Cistus species (Cistaceae). Botanical Journal of the Linnean Society, 109(1): 39-55.

BRANDT, U. \& G. GOTTSBER GER -1988- Flower phenology, pollinating insects and breeding systems in Cistus, Halimium and Tuberaria species in Portugal. Lagascalia, 15: 625-634.

CORBET, S.A. -1978- Bee visits and the nectar of Echium vulgare L. and Sinapis alba L. Ecol. Entomol., 3: 25-27. 
CORBET, S.A., C.J.C. KERSLAKE, D. BROWN y N.E. MORLAND -1984- Can bees select nectarrich flowers in patch?. J. Apic. Res., 23(4): 234242.

CORBET, S.A., D.M. UNWIN y O.E. PRYS-JONES $-1979 a-$ Humidity, nectar and insect visits to flowers, with special reference to Crataegus, Tilia and Echium. Ecol. Entomol., 4: 9-22.

CORBET, S.A., P.G. WILLMER, J.W.L. BEAMENT, D.M. UNWIN y O.E. PRYS-JONES $-1979 b$ - Post-secretory determinants of sugar concentration in nectar. Plant Cell and Environment, 2: 293-308.

CORBET, S.A., P.G. WILLMER, J.W.L. BEAMENT, D.M. UNWIN y O.E. PRYS-JONES -1979 b- Post-secretory determinants of sugar concentration in nectar. Plant Cell and Environment, 2: 293-308.

CRUDEN, R.W. y S.M. HERMANN -1983Studying nectar? Some observations on the art. En B. BENTLEY y T. ELIAS (eds.). The biology of nectaries., Columbia University Press. New York.

DEVESA, J.A., J. ARROYO y J. HERRERA -1985Contribución al conocimiento de la biología floral del género Lavandula L. Anales Jard. Bot. Madrid, 42(1): 165-186.

FERNANDEZ, C., A. MUÑOZ y J.A. DEVESA -1989- Las digitalis extremeñas. Vida apicola, 35: 27-33.

HERRERA, J. -1985a-Biología reproductiva del matorral de Doñana. Tesis Doctoral. Universidad de Sevilla.

HERRERA, J. -1985b- Nectar secretion patterns in southern spanish mediterranean shrublands. Israel J. Bot., 34: 47-58.

HERRERA, J. -1987a- Biología reproductiva de algunas especies del matorral de Doñana. Anales Jard. Bot. Madrid, 44(2): 483-497.

HIDALGO, M.I y B. CABEZUDO -1994- Fenología y volumen de floración del matorral de la Sierra de Mijas (Málaga, SE España). Acta Bot. Malacitana, 19: 123-136.

HIDALGO, M.I. y B. CABEZUDO -1995Producción floral en un matorral del S de España como base para su valoración melitopalinológica. Anales Jard. Bot Madrid, (en prensa).

INOUYE, D.W., N.D. FAVRE, J.A. LANUN, D.M. LEVINE, J.B. MEYERS, M.S. ROBERTS, F.C.
TSAO y Y.Y. WANG -1980- The effects of nonsugar nectar constituents on estimates of nectar energy content. Ecology, 61(4): 992-997.

MUÑOZ, A. y J.A DEVESA -1987- Contribución al conocimiento de la biología floral del género Lavandula L. II. Lavandula stoechas L. subsp. stoechas. Anales Jard. Bot. Madrid, 44(1): 6378.

NUÑEZ, J. -1977- Nectar flow by melliferous flora and gathering flow by Apis mellifera ligustica. J. Insect Physiol., 23: 265-275.

ORTIZ, P. -1991- Melitopalinología en Andalucía Occidental. Tesis Doctoral. Universidad de Sevilla.

ORTIZ, P.L. -1994- The Cistaceae as food resources for honey bees in SW Spain. Journ. Apic. Research, 33(3): 136-144.

PERCIVAL, M. -1961- Types of nectar in Angiospermas. New Phytol., 60: 235-281.

PERCIVAL, M. -1961- Types of nectar in Angiospermas. New Phytol., 60: 235-281.

PERCIVAL, M. -1961- Types of nectar in Angiospermas. New Phytol., 60: 235-281.

PFLUMM, W. -1985- Influence of nectar-supply rate on the number of flowers visited by a honeybee on each collecting flight. Oecologia, 66: 207-210.

SHUEL, R.W. -1975- La producción de néctar. In DADANT et al. (eds) La colmena y la abeja melífera. Hemisferio Sur. Montevideo.

TALA VERA, S., J. HERRERA, J. ARROYO, P.L. ORTIZ y J.A. DEVESA -1988- Estudio de la flora apícola de Andalucía Occidental. Lagascalia, 15(extra): 567-591.

TALAVERA, S., P.E. GIBBS \& J. HERRERA 1993-Reproductive biology of Cistus ladanifer (Cistaceae) Pl. Syst. Ecol., 186: 123-134.

WEBER EL-GHOBARY, M.O. -1984-Observation of flowering, pollen, nectar and pollen loads, due to Apis mellifera in some mediterranean plants. Les colloques de e'INRA, 21: 245-250.

WILLMER, P.G. -1980- The effects of insect visitor on nectar constituents in temperature plants. Oecologia, 47: 270-277.

Aceptado para su publicación en Enero de 1995

Dirección de los autores. Dpto. Biología Vegetal. Facultad de Ciencias. Universidad de Málaga. Campus de Teatinos. Apdo. 59. 29080, Málaga. Fax. (95) 2131944 